ACT-03/95

CERN-TH.7480/94

CTP-TAMU-12/95

ENSLAPP-A.492/94

\title{
A String Scenario for Inflationary Cosmology
}

\author{
John Ellis $^{a}$, N.E. Mavromatos ${ }^{b, \diamond}$ and D.V. Nanopoulos ${ }^{a, c}$
}

\begin{abstract}
We describe a scenario for inflation in the framework of non-critical string theory, which does not employ an inflaton field. There is an exponential expansion of the volume of the Universe, induced by enormous entropy production in the early stages of cosmological evolution. This is associated with the loss of information carried by global string modes that cross the particle horizon. It is the same loss of information that induces irreversible time flow when target time is identified with the worldsheet Liouville mode. The resulting scenario for inflation is described by a string analogue of the Fokker-Planck equation that incorporates diffusion and dissipative effects. Cosmological density perturbations are naturally small.

${ }^{a}$ Theory Division, CERN, CH-1211, Geneva, Switzerland,

${ }^{b}$ Laboratoire de Physique Thèorique ENSLAPP (URA 14-36 du CNRS, associeè à l' E.N.S de Lyon, et au LAPP (IN2P3-CNRS) d'Annecy-le-Vieux), Chemin de Bellevue, BP 110, F-74941 Annecy-le-Vieux Cedex, France ;

$\diamond$ On leave from P.P.A.R.C. Advanced Fellowship, Dept. of Physics (Theoretical Physics), University of Oxford, 1 Keble Road, Oxford OX1 3NP, U.K.

${ }^{c}$ Center for Theoretical Physics, Dept. of Physics, Texas A \& M University, College Station, TX 77843-4242, USA and Astroparticle Physics Group, Houston Advanced Research Center (HARC), The Mitchell Campus, Woodlands, TX 77381, USA.
\end{abstract}

ACT-03/95

CERN-TH.7480/94

CTP-TAMU-12/95

ENSLAPP-A.492/94

March 1995 


\section{Introduction}

Inflation [1, 2] offers an attractive solution to many of the major problems of conventional Big Bang cosmology, such as the age, size and homogeneity of the observed Universe, its large entropy and its closeness to the critical density. Inflation occurs for generic scalar field potentials, but these may not fit naturally within a conventional Grand Unified field theory. A number of proposals have been made

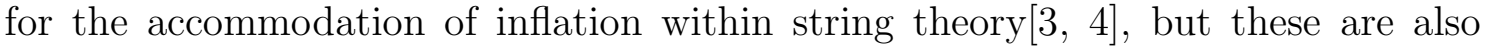
beset with difficulties. At the level of an effective field theory derived from an underlying string theory, it becomes even more difficult to generate naturally a suitable inflationary scalar potential. Some such scenaria have inflationary epochs that are too short and/or inhomogeneous density fluctuations that are too large 3 . However, we do not rule out the possibility that a realistic scenario may emerge under certain extra assumptions.

A more fundamental approach has been to study full string theories in timedependent backgrounds [5], in the hope of identifying string solutions of cosmological interest that could serve as the basis of an inflationary model. Consistent partition functions for such cosmological string theories have been constructed, featuring a dilaton field that depends linearly on time. This is linked to a deficit $Q^{2}$ in the central charge that is compensated by other degrees of freedom to restore criticality. Matter fields move in such a non-critical time-dependent background according to equations of motion containing extra 'frictional' terms $\propto Q$, which plays the rôle of the Hubble expansion parameter, and the time-dependent dilaton can be identified as a non-critical Liouville field, as we discuss in more detail later. Such cosmological string models do not exhibit exponential inflation, when treated at the classical level[5], but we recall that the quantum effects play essential rôles in conventional field-theoretical inflation [1, 2], e.g. in providing initial fluctuations and in converting scalar field energy into matter-particle entropy [6]. We are therefore led to seek a consistent quantum treatment of time-dependent string theories, which provides the string scenario for inflation discussed below.

We first review salient features of conventional field-theoretical inflation, emphasizing a frictional interpretation of the Hubble expansion and the relation of entropy generation to the integration over field degrees of freedom that disappear beyond the de Sitter horizon. Then we construct a string analogue starting from the Zamolodchikov function interpreted as an effective action in string theory space [7, 8], demonstrating that it leads to a consistent quantum formulation that incorporates dissipation, associated with couplings to unobserved string modes. The previous cosmological string theories [5] can be regarded as specific examples of this general framework. In the cosmological context, the dissipation is related to global string modes [9] that cannot be detected via local experiments within a horizon volume. Then we derive a string analogue of the Fokker-Planck equation, demonstrate that it may lead to the classical 'ball rolling down the hill' picture of conventional field 
theoretical inflation [1, 2], as well as exponential increases in the cosmological scale factor and matter-particle entropy [6]. This inflation is achieved without an inflaton field and can be regarded as a world-sheet analogue of Starobinski's ideas [10]. The magnitude of density perturbations is related to the ratio of particle and higher string mode energies, and is naturally small.

\section{Review of Field-Theoretical Inflation}

In conventional field-theoretical inflation [1, 2], the (near-) exponential expansion of the Universe is driven by the potential energy $V(\Phi)$ of a specially-introduced inflaton field $\Phi$, treated classically $\Phi \simeq \Phi_{c}$ as a first approximation, evolving according to the equation

$$
\nabla^{2} \Phi_{c}+\left.\frac{\delta}{\delta \Phi_{c}} V(\Phi)\right|_{\Phi=\Phi_{c}}=0
$$

Assuming spatial homogeneity, inserting a Robertson-Walker-Friedmann scale factor of $e^{H t}$ into the first term in (1), and approximating the second term by $-\mu^{2} \Phi_{c}$, one obtains

$$
\ddot{\Phi}_{c}+3 H \dot{\Phi}_{c}-\mu^{2} \Phi_{c}=0
$$

This equation exhibits frictional dissipation $\propto H$. In the interesting limit of a slow rollover, the growing solution of (2) has

$$
\Phi_{c} \simeq \exp \left(\frac{\mu^{2}}{3 H} t\right)
$$

yielding a typical rollover time $\tau \simeq \frac{3 H}{\mu^{2}}$ and an exponential growth in the scale factor by an amount

$$
R / R_{0} \simeq \exp (H \tau) \simeq e^{\frac{3 H^{2}}{\mu^{2}}}
$$

This is sufficiently large $(H \tau \geq 60)$ if the effective potential $V\left(\Phi_{c}\right)$ is sufficiently flat, i.e.

$$
3 H^{2} \simeq 8 \pi G_{N} V\left(\Phi_{c}\right) \geq 60 \mu^{2} \simeq 60 V^{\prime \prime}\left(\Phi_{c}\right)
$$

An order-of-magnitude lower bound on $\mu^{2}$ is provided by the Hawking temperature $T_{H}=\frac{H}{2 \pi}$ associated with the presence of a horizon during the de-Sitter-like inflationary epoch. This limits the roll-over-time $\tau \leq 3 H / T_{H}^{2}$, so that the scale factor factor grows by a factor of at most $\exp \left(12 \pi^{2}\right)$, which is ample to accommodate our observable Universe.

The finite temperature $T_{H}$ is related to the area $A=12 \pi / H^{2}$ of the de Sitter horizon, in close parallelism to the Hawking temperature of a black hole horizon. In each case, information is lost across the horizon, as a consequence of the necessary integration over unseen field modes beyond the horizon. In the inflationary case, the 
entropy per horizon volume $S_{H} \propto A$, and the total entropy grows exponentially along with the scale factor (14). This can be regarded as due to the separation of the field modes into two categories, only one of which, $\Phi_{<}$, is inside the horizon and hence observable, whilst the other acts as a stochastic noise source in an application of the fluctuation-dissipation theorem to the observable modes. A stochastic framework has been suggested for inflation, which is based on the following Fokker-Planck equation for the probability distribution $\mathcal{P}\left(\Phi_{<}, t\right)$ of the observable modes [2, 11]

$$
\partial_{t} \mathcal{P}\left(\Phi_{<, t}\right)=\frac{1}{8 \pi^{2}} \frac{\delta}{\delta \Phi_{<}}\left(H^{\frac{3}{2}} \frac{\delta}{\delta \Phi_{<}}\left[H^{\frac{3}{2}} \mathcal{P}\left(\Phi_{<}, t\right)\right]\right)+\frac{1}{6} \frac{\delta}{\delta \Phi_{<}}\left[\frac{1}{H} \frac{\delta}{\delta \Phi_{<}} V\left(\Phi_{<}\right) \mathcal{P}\left(\Phi_{<, t}\right)\right]
$$

where the standard deviation of the observable modes is identified with the zeropoint fluctuation of a massless scalar field in a de Sitter background,

$$
<\left(\delta \Phi_{<}\right)^{2}>=\frac{1}{4 \pi^{2}} H^{3} \delta t
$$

Such density fluctuations in the observable Universe may be regarded as thermal fluctuations associated with the finite horizon.

In the above brief review, we have glossed over the transition from the initial quantum regime to the classical 'ball rolling down the hill' picture (11), and it is desirable to establish a unified description of this, entropy generation during inflationary epoch and the conversion into matter particles at the end of inflation. Given the progress in this direction made in conventional field-theoretic inflation [1], we now seek a corresponding framework in string theory.

\section{String Framework}

Our starting point is the recognition that a suitable variant of the Zamolodchikov [7] $C$ function $C\left[g^{i}\right]$ can be regarded as an effective action in the space of two-dimensional field theories on the world-sheet, parametrized by the couplings $g^{i}$ of generalized $\sigma$ models

$$
\begin{aligned}
C[g]= & \int d^{2} z\left(2 z^{4}<T_{z z}(z, \bar{z}) T_{z z}(0,0)>_{g}-3 z^{3} \bar{z}<T_{z z}(z, \bar{z}) T_{z \bar{z}}(0,0)>_{g}-\right. \\
& \left.6 z^{2} \bar{z}^{2}<T_{z \bar{z}}(z, \bar{z}) \Theta(0,0)>_{g}\right)
\end{aligned}
$$

where $T_{\alpha \beta}$ are components of the world-sheet stress tensor, whose trace is $\Theta$. The couplings $g^{i}$ correspond in general to non-conformal, relevant deformations $V_{i}$, with anomalous dimensions $h_{i}<2$ and non-trivial flow under the renormalization group, which is described by

$$
\beta^{i} \equiv \frac{d}{d l n \mu} g^{i}=\left(h_{i}-2\right) g^{i}-\pi c_{j k}^{i} g^{j} g^{k}
$$


where $\mu$ is a renormalization group scale and the $c_{j k}^{i}$ are operator-product-expansion coefficients for the $V_{i}$. The corresponding classical equations of motion are the familiar gradient flow

$$
G_{i j} \beta^{j}=\frac{\delta C}{\delta g^{i}}
$$

where $G_{i j}=<V_{i} V_{j}>$ is the metric in coupling space.

Coupling (8) to two-dimensional quantum gravity restores [5, 12 conformal invariance at the quantum level, by dressing the vertex operators : $V_{i} \rightarrow\left[V_{i}\right]_{\phi}$, where $\phi$ is the Liouville field that scales the world-sheet metric $\gamma_{\alpha \beta}=e^{\phi(z, \bar{z})} \hat{\gamma}_{\alpha \beta}$ where $\hat{\gamma}$ denotes a fixed fiducial metric. In our approach, $\phi(z, \bar{z})$ serves as a local renormalization scale on the world sheet. The dressed vertex operators $\left[V_{i}\right]_{\phi}$ are then exactly marginal and the corresponding gravitationally-renormalized couplings are [12, 13]

$$
\lambda^{i}(\phi)=g^{i} e^{\alpha^{i} \phi}+\frac{\pi}{Q \pm 2 \alpha^{i}} c_{j k}^{i} g^{j} g^{k} \phi e^{\alpha_{i} \phi}+\ldots
$$

where [12

$$
Q=\sqrt{\frac{|25-c|}{3}+\frac{1}{4} \beta^{i} G_{i j} \beta^{j}} \quad ; \quad \alpha_{i}^{2}+\alpha_{i} Q=\operatorname{sgn}(25-c)\left(h_{i}-2\right)
$$

is the central charge deficit of the matter theory defined by $g^{i}$, and the appropriate gravitationally-dressed version of (8) is supplemented by the Liouville action

$$
\begin{aligned}
S_{m L} & =S_{m}+S_{L} \\
S_{L} & =\frac{1}{4 \pi \alpha^{\prime}} \int d^{2} z \sqrt{\hat{\gamma}}\left[\left(\partial_{\alpha} \phi\right)\left(\partial^{\alpha} \phi\right)-Q \phi R^{(2)}\right] \\
S_{m} & =\int d^{2} z \sqrt{\hat{\gamma}} \lambda^{i}(\phi) V_{i}
\end{aligned}
$$

where $R^{(2)}$ is the curvature of the world-sheet. Note that we normalize the Liouville field so as to have a canonical kinetic term on the world sheet, enabling us to identify $\phi$ with the target time, which we denote by $t$. This will be the physical time, observed in standard units. However, for reasons that will become clear later on, we introduce a rescaled time $\tau$ :

$$
\phi \equiv t=Q \tau
$$

We shall come back to the physical time $\phi=t$ when we discuss our estimates for physical observables. The matter system is supercritical if $c>25$, a possibility first studied as a prototype for an expanding Universe in the framework of string theory in ref. [5], whose 'physical time' is $e^{Q \phi}$ in our notation. Equation (12) has the solutions

$$
\alpha_{i}^{ \pm}=-\frac{Q}{2} \pm \sqrt{Q^{2}-\left(h_{i}-2\right)} \quad ; \quad c \geq 25
$$

The branch of solutions $\alpha_{i}^{-}$corresponds to non-existing operators in Liouville theory with the wrong boundary conditions [14], and should be excluded. This leaves us with the physically-relevant solutions $\alpha_{i}^{(+)}$, which vanish for $(1,1)$ operators, which are made exactly marginal by the linear factor in the second term of (111). 
In ref. [9] we went further than in [5], identifying the Liouville field $\phi=Q \tau$ with a world-sheet renormalization scale via the world-sheet area

$$
A \equiv \int d^{2} z \sqrt{\hat{\gamma}} e^{\alpha \phi(z, \bar{z})} \quad ; \quad \alpha=-\frac{Q}{2}+\frac{1}{2} \sqrt{Q^{2} \pm 8}
$$

Equation (14) implies that derivatives with respect to our target time variable $t$ of the Liouville-dressed $(1,1)$ couplings in the neighborhood of a fixed point are simply the conventional $\beta$ functions (9) with the replacements $g^{i} \rightarrow \lambda^{i}$ :

$$
\dot{\lambda}^{i} \equiv \frac{d \lambda^{i}}{d \tau}=\beta^{i}(\lambda)
$$

which makes a Liouville flow similar (formally) to a conventional renormalization group flow on the world sheet. Here and subsequently, derivatives with respect to $\tau$ are denoted by dots. In this formalism, the deficit $Q$, which is a constant at lowest order in the coupling, becomes (12) a 'running' central charge that approaches a constant at a fixed point. This renormalization group flow may be interpreted as a friction problem [9] with dissipation coefficient $Q$, since $(1,1)$ deformations $\lambda^{i}(\tau)$ obey the equation [15]

$$
\ddot{\lambda}^{i}(\tau)+Q^{2} \dot{\lambda}^{i}(\tau)=-Q^{2} \beta^{i}(\lambda)=-Q^{2} \dot{\lambda}^{i}=-Q^{2} G^{i j} \frac{\partial}{\partial \lambda^{j}} C(\lambda)
$$

Not only is this equation characteristic of frictional motion in a potential $C\left(\lambda^{i}\right)$, (10), but it is similar to the inflaton equation (1, 2). Upon the inverse rescaling (14), the friction term becomes $\propto Q$, which implies that in standard units the Hubble constant

$$
H=\frac{Q}{3}
$$

The pioneering cosmological solution of [5] can be regarded as a special case of (18), in which the running of $Q$ was not considered. As was pointed out in [5], the appearance of a central charge deficit $Q^{2}$ is accompanied by a non-trivial dilaton potential, whose magnitude determines the Hubble expansion rate.

Our discussion of a string inflationary scenario requires the appropriate generalization 9 of the dissipative equation (18) to the statistical (equilibrium) distribution function in string theory space $\rho\left(\lambda^{i}, p_{j}, \tau\right)$. The $p_{i}$ denote momenta conjugate to the 'coordinates' $\lambda^{i}$, whose expectation values are identified with the pertinent vertex operators $V_{i}$. The renormalizability of the world-sheet $\sigma$-model tells us that

$$
\begin{aligned}
\mathcal{D} S_{m L} & =0 \\
\mathcal{D} \equiv \frac{d}{d \tau}=\partial_{\tau}+\dot{\lambda}^{i} \partial_{i}+\dot{p}_{i} \partial_{p_{i}} & =\partial_{\tau}+\beta^{i} \partial_{i}+\dot{p}_{i} \partial_{p_{i}}
\end{aligned}
$$

and in particular that

$$
\mathcal{D} \rho=0=\frac{\partial}{\partial \tau} \rho+\beta^{i} \frac{\partial}{\partial \lambda^{i}} \rho+\dot{p}^{i} \frac{\partial}{\partial p_{i}} \rho
$$


Substituting in (21) using the classical equations of motion derivable from (8) :

$$
\dot{\lambda}^{i}=\beta^{i}=\frac{\partial}{\partial p_{i}} \mathcal{H} \quad ; \quad \dot{p}_{i}=-\frac{\partial}{\partial \lambda^{i}} \mathcal{H}-G_{i j} \beta^{j}
$$

we derive 9

$$
\frac{\partial}{\partial \tau} \rho=-\{\rho, \mathcal{H}\}-\beta^{i} G_{i j} \frac{\partial \rho}{\partial p_{j}}
$$

where $\{$,$\} denotes the conventional Poisson bracket, reflecting the fact that we are$ still working at the classical level. The second term in (23) represents dissipation 16] due to couplings with unobservable modes.

\section{Quantum Consistency}

As is well known, higher-genus effects in string theory impose quantization of the effective string couplings $\lambda^{i}$ [17. Since our goal is a full quantum description of the

string inflation problem, we must check that the equations (18) are consistent with canonical quantization:

$$
\left[\lambda^{i}, \lambda^{j}\right]=0 \quad ; \quad\left[p_{i}, p_{j}\right]=0 \quad ; \quad\left[\lambda^{i}, p_{j}\right]=-i \hbar \delta_{j}^{i}
$$

This is true if there is an underlying Lagrangian $L$ [18] whose equations of motion are equivalent but not necessarily identical to (18), i.e. if there exists a non-singular matrix $w_{i j}$ :

$$
w_{i j}\left(\ddot{\lambda}^{j}+2 Q^{2} \dot{\lambda}^{j}\right)=\frac{d}{d \tau}\left(\frac{\partial L}{\partial \dot{\lambda}^{i}}\right)-\frac{\partial L}{\partial \lambda^{i}}
$$

which obeys the following Helmholtz conditions :

$$
\begin{aligned}
w_{i j} & =w_{j i} \\
\frac{\partial w_{i j}}{\partial \dot{\lambda}^{k}} & =\frac{\partial w_{i k}}{\partial \dot{\lambda}^{j}} \\
\frac{1}{2} \frac{D}{D \tau}\left(w_{i k} \frac{\partial f^{k}}{\partial \dot{\lambda}^{j}}-w_{j k} \frac{\partial f^{k}}{\partial \dot{\lambda}^{i}}\right) & =w_{i k} \frac{\partial f^{k}}{\partial \lambda^{j}}-w_{j k} \frac{\partial f^{k}}{\partial \lambda^{i}} \\
\frac{D}{D \tau} w_{i j} & =-\frac{1}{2} w_{i k} \frac{\partial f^{k}}{\partial \dot{\lambda}^{j}}-\frac{1}{2} w_{j k} \frac{\partial f^{k}}{\partial \dot{\lambda}^{i}}
\end{aligned}
$$

where

$$
f^{i} \equiv-2 Q^{2} \dot{\lambda}^{i} \quad ; \quad \frac{D}{D \tau} \equiv \partial_{\tau}+\dot{\lambda}^{i} \partial_{i}+f^{i} \frac{\partial}{\partial \dot{\lambda}^{i}}
$$

If the conditions (26) are met, then 18

$$
w_{i j}=\frac{\partial^{2} L}{\partial \dot{\lambda}^{i} \partial \dot{\lambda}^{j}}
$$


The Lagrangian in (28) can be determined up to total derivatives according to [16, 18]:

$\mathcal{S} \equiv \int d \tau L=-\int d \tau \int_{0}^{1} d \kappa \lambda^{i} E_{i}(\tau, \kappa \lambda, \kappa \dot{\lambda}, \kappa \ddot{\lambda}) ; E_{i}(\tau, \lambda, \dot{\lambda}, \ddot{\lambda}) \equiv w_{i j}\left(\ddot{\lambda}^{j}+Q^{2} \dot{\lambda}^{i}+Q^{2} \beta^{i}\right)$

In our case, near a fixed point where the variation in $Q$ can be neglected, up to $O\left[\beta^{3}\right]$ it acquires the form

$$
\mathcal{S}=-\frac{1}{2} \int d \tau\left(\dot{\lambda}^{i} G_{i j}[\lambda, \tau] \dot{\lambda}^{j}+\ldots\right)
$$

with the ... denoting terms that can be removed by a renormalization scheme change. Within a critical-string (on-shell) approach, the action (29,30) can be considered as an effective action generating the string scattering amplitudes. Here it should be considered as a target-space 'off-shell' action [8] for non-critical strings. Comparing (30) with equation (28), and taking into account the renormalization group invariance of $L$, we see immediately that one should identify $w_{i j}=-G_{i j}$, the metric in coupling space.

We know that $G_{i j}$ is symmetric, so the first of the Helmholtz conditions (26) is satisfied. The next two conditions hold automatically because of the gradient flow property (10) and the fact that $G_{i j}$ and $C(8)$ are functions of the coordinates $\lambda^{i}$ and not of the conjugate momenta. Finally, the fourth Helmholtz condition provides the condition

$$
\frac{D}{D \tau} G_{i j}=2 Q^{2} G_{i j}
$$

which implies an expanding scale factor for the metric in string theory space

$$
G_{i j}[\tau, \lambda(\tau)]=e^{2 Q^{2} \tau} \hat{G}_{i j}[\tau, \lambda(\tau)]
$$

where $\hat{G}_{i j}$ is a Liouville-renormalization-group invariant function. This is exactly the form of the Zamolodchikov metric in Liouville strings [19]. Thus there is indeed an underlying Lagrangian, one can quantize consistently, and

$$
\partial_{\tau} \rho=i[\rho, \mathcal{H}]+i \beta^{j} G_{j i}\left[\lambda^{i}, \rho\right]
$$

is the appropriate quantum version of the density matrix equation (23).

It is straightforward that the observable energy $E=<<\mathcal{H}>>=\operatorname{Tr}(\rho \mathcal{H})$ is conserved in this approach 20], as a consequence of the renormalizability of the underlying two-dimensional field-theoretic formalism,

$$
\begin{aligned}
\partial_{\tau}<<\mathcal{H}>> & =<<\partial_{\tau} C+\partial_{\tau} \mathcal{H}>>= \\
=\partial_{\tau}<<C>> & +<<\partial_{\tau} \mathcal{H}>>\propto \partial_{\tau}<<\partial_{i} \beta^{j}>>=0
\end{aligned}
$$


where one took into account that $C$ is a renormalization- group-invariant functional of $\lambda^{i}$ only. However, the quantum energy fluctuations $\delta E \equiv\left[<<\mathcal{H}^{2}>>-(<<\right.$ $\left.\mathcal{H}>>)^{2}\right]^{\frac{1}{2}}$ are time-dependent :

$$
\partial_{\tau}(\delta E)^{2}=-i<<\left[\beta^{i}, \mathcal{H}\right] \beta^{j} G_{j i}>>=<<\beta^{j} G_{j i} \frac{d}{d \tau} \beta^{i}>>
$$

Using the fact that $\beta^{i} G_{i j} \beta^{j}$ is a renormalization-group invariant quantity, we can express (35) in the form

$$
\partial_{\tau}(\delta E)^{2}=-<<Q^{2} \beta^{i} G_{i j} \beta^{j}>>=-<<Q^{2} \partial_{\tau} C>>
$$

where equation (31) has been taken into account. This is the basis for the estimate we present later of the quantum density fluctuations generated during the string inflationary epoch.

\section{$5 \quad$ String Fokker-Planck Equation}

Having established the canonical quantization of the dynamics in string theory space, we now sketch the derivation of the string Fokker-Planck equation that incorporates quantum effects in the renormalization group flow equation (18). We interpret this as an equation for the classical coupling $\lambda_{c}(\tau)$, and decompose the value $\lambda_{c}(\tau+\delta \tau)$ of the coupling at an infinitesimally later time as

$$
\lambda_{c}^{i}(\tau+\delta \tau)=\lambda_{c}^{i}(\tau)+\dot{\lambda}_{c}^{i}(\tau) \delta \tau+\delta \lambda_{q}^{i}(\delta \tau)
$$

where $\delta \lambda_{q}(\tau)$ is the quantum fluctuation. We assume that this has a characteristic white-noise distribution

$$
P\left(\delta \lambda_{q}\right)=\left[\frac{1}{2 \pi<\delta \lambda_{q}>^{2}}\right]^{\frac{1}{2}} \exp \left(-\frac{\left(\delta \lambda_{q}\right)^{2}}{2<\left(\delta \lambda_{q}\right)^{2}>}\right)
$$

arising from unobservable global string modes that extend beyond the horizon. This assumption can be justified in standard field-theoretic inflation by an analysis of quantum fluctuations in de Sitter space [2, 11]. The analogue in our case is

$$
<\left(\delta \lambda_{q}\right)^{2}>=\frac{Q^{6}}{4 \pi^{2}} \delta \tau
$$

which we will justify further a posteriori. White noise in renormalization group flow has been assumed previously [21, by analogy with the conventional Langevin equation (gradient flow form of the $\beta$-functions [7]). In our interpretation this random noise is induced by the sum over unobservable string modes beyond the event horizon, whose effects are captured in our approach by the Liouville time field and its quantum fluctuations. 
It is straightforward to derive the string Fokker-Planck equation from (18, 37, 38, 39). The probability distribution $\mathcal{P}(\lambda, \tau)$ in string coupling space may be represented as as

$$
\mathcal{P}(\lambda, \tau)=\int d \lambda_{c} P\left(\delta \lambda_{q}\right) \mathcal{P}\left(\lambda_{c}, \tau\right)
$$

where the integral is over all classical variables $\lambda_{c}$ whose evolution in time yields the variable $\lambda$ at rescaled time $\tau$. Changing variables to the quantum fluctuations $\delta \lambda_{q}$, we find

$$
\mathcal{P}(\lambda, \tau)=\int d\left(\delta \lambda_{q}\right) \mathcal{P}\left(\lambda^{i}-\partial^{i} C(\lambda) \delta \tau-\delta \lambda_{q}^{i}\right) \operatorname{det}\left(1+\frac{\partial}{\partial \lambda}\left(\partial_{\lambda} C(\lambda)\right)-\partial_{\lambda}\left(\delta \lambda_{q}\right)\right)
$$

It is a simple matter to make a Taylor expansion [11] of the integrand in (41) and perform the Gaussian integral over $\delta \lambda_{q}$ to obtain the string Fokker-Planck equation

$$
\partial_{\tau} \mathcal{P}(\lambda, \tau)=\frac{1}{8 \pi^{2}} \frac{\delta}{\delta \lambda^{i}} Q^{3} \delta^{i j} \frac{\delta}{\delta \lambda^{j}}\left[Q^{3} \mathcal{P}(\lambda, \tau)\right]+\frac{\delta}{\delta \lambda^{i}}\left[\beta^{i} \mathcal{P}(\lambda, \tau)\right]
$$

modulo ordering ambiguities for the $\lambda$-dependent diffusion coefficients. The reader may verify that the dispersion $\left\langle\left(\delta \lambda_{q}\right)^{2}>\right.$ calculated from (42) is of the assumed form (39)円. Equation (42) may alternatively be derived [19 from equation (33) for the statistical distribution in string theory space, $\left\{\lambda^{i}\right\}$, if one assumes

$$
\rho \simeq e^{-\overline{\mathcal{H}}} W\left(\lambda^{i}, \tau\right)
$$

as in the conventional 22] treatment of Markov processes, where $\overline{\mathcal{H}} \equiv-\frac{8 \pi^{2} \mathcal{H}}{Q^{6}}$ with $Q^{6} / 8 \pi^{2}$ playing the rôle of an effective 'temperature' in theory space.

The string Fokker-Planck equation (42) can be used to demonstrate that the probability distribution function $\mathcal{P}$ takes the Gibbs form [23] $\mathcal{P}=e^{-\overline{\mathcal{H}}}$ asymptotically at large times, i.e. that $W(\lambda, \tau) \rightarrow 1$ as $\tau \rightarrow \infty$. This is done by first noting that the non-self-adjoint first-derivative term in (42) can be written in the form

$$
\frac{\partial}{\partial \lambda^{i}}\left(\beta^{i} \mathcal{P}(\lambda, \tau)\right)=-\frac{\partial}{\partial \lambda^{i}}\left(G^{i j} \frac{\partial \mathcal{H}}{\partial \lambda^{j}} \mathcal{P}(\lambda, \tau)\right)
$$

using the fact that $\dot{p}_{i}=0$ in our scheme. The quantity $\hat{\mathcal{P}}\left(\lambda^{i}, \tau\right) \equiv e^{\frac{1}{2} \overline{\mathcal{H}}} \mathcal{P}\left(\lambda^{i}, \tau\right)$ therefore obeys the equation

$$
\hat{O}_{F P} \hat{\mathcal{P}}\left(\lambda^{i}, \tau\right)=0 ; \quad \hat{O}_{F P} \equiv-\frac{Q^{6}}{8 \pi^{2}}\left[-\frac{\partial}{\partial \lambda^{i}}+\frac{4 \pi^{2}}{Q^{6}} \frac{\partial \mathcal{H}}{\partial \lambda^{i}}\right]\left[\frac{\partial}{\partial \lambda^{i}}+\frac{4 \pi^{2}}{Q^{6}} \frac{\partial \mathcal{H}}{\partial \lambda^{i}}\right]
$$

where $\hat{O}_{F P}$ is self-adjoint. Thus we can expand

$$
\mathcal{P}\left(\lambda^{i}, t\right)=e^{-\frac{1}{2} \overline{\mathcal{H}}} \sum_{n} a_{n} e^{\varepsilon_{n} t} f_{n}\left(\lambda^{i}\right)
$$

\footnotetext{
${ }^{1}$ Energy conservation (34) can also be derived using the Fokker-Planck equation, when one takes into account restrictions on the probability distributions that are consequences of the renormalization group.
} 
where the $f_{n}\left(\lambda^{i}\right)$ are eigenfunctions of $\hat{O}_{F P}$ with negative semi-definite eigenvalues $\varepsilon_{n}$ :

$$
\varepsilon_{n}=\int d \lambda^{i} f_{n}^{*} \hat{O}_{F P} f_{n}=-\frac{Q^{6}}{8 \pi^{2}} \int d \lambda^{i}\left|\left(\frac{\partial}{\partial \lambda^{i}}-\frac{4 \pi^{2}}{Q^{6}} \frac{\partial \mathcal{H}}{\partial \lambda^{i}}\right) f_{n}\right|^{2} \leq 0
$$

where $\varepsilon_{0}=0$ for $f_{0}=e^{-\frac{1}{2} \overline{\mathcal{H}}}$, and the contraction of the indices is done with the help of the positive definite Zamolodchikov metric $G_{i j}$. It follows that $\mathcal{P}\left(\lambda^{i}, t\right) \rightarrow a_{0} e^{-\overline{\mathcal{H}}}$, the equilibrium Gibbs distribution, at large times ??

\section{Realization of Inflation}

The string Fokker-Planck equation (42) can be used to discuss the circumstances under which the entropy

$$
S=-k_{B} \int\left[D \lambda^{i}\right] \mathcal{P}(\lambda, \tau) \ln \mathcal{P}(\lambda, \tau)
$$

may grow exponentially, as in inflationary cosmology. Using (42), we find

$$
\partial_{\tau} S=\int\left[D \lambda^{i}\right] \frac{k_{B}}{2}\left[\frac{Q^{6}}{4 \pi^{2}} \frac{\left|\nabla_{i} \mathcal{P}\right|^{2}}{\mathcal{P}}+\frac{1}{8 \pi^{2}}\left(\nabla_{i} Q^{6}\right) \nabla^{i} \mathcal{P}+2 \beta^{i} \nabla_{i} \mathcal{P}\right]
$$

As a result of the renormalization-group invariance of $\mathcal{P}$, the last term on the righthand side of (49) contributes boundary terms in string theory space, which we discard in our cosmological context. We assume that the second term $\propto \nabla^{i} Q^{6}$ is much smaller than the first term, corresponding to an inflationary epoch during which $Q$ is approximately constant, obtaining

$$
\partial_{\tau} S=\int\left[D \lambda^{i}\right] \frac{k_{B}}{8 \pi^{2}}\left[Q^{6} \frac{\left|\nabla_{i} \mathcal{P}\right|^{2}}{\mathcal{P}}\right] \geq 0
$$

The positivity of (50) is a consequence of world-sheet unitarity, and the zero occurs only at fixed points where $Q \rightarrow 0$. Using now the Gibbs formula which implies that $\ln \mathcal{P}=-\overline{\mathcal{H}}=\frac{8 \pi^{2} \mathcal{H}}{Q^{6}}$, and recalling that $\nabla^{i} \mathcal{H} \simeq-\beta^{i}$, we see that

$$
\dot{S}=-k_{B} \int[D \lambda] \frac{1}{|\mathcal{H}|}\left(\beta^{i} \beta_{i}\right) \mathcal{P} \ln \mathcal{P}
$$

It is apparent that if $\beta^{i} \beta_{i} \simeq Q^{4}$ varies sufficiently slowly over a sufficiently long period of time - the string analogue of the "slow rollover" assumption of conventional inflation [2, 11] - the entropy will indeed grow exponentially :

$$
S \propto e^{Q^{2} \tau}=e^{Q t}
$$

since $\mathcal{H}$ is of $O\left[Q^{2}\right]$ during inflation. The re-expression of (52) in terms of the physical time $t$ confirms the correspondence (19) of the central charge deficit $Q$ to the conventional Hubble expansion rate during inflation.

\footnotetext{
${ }^{2}$ This result has been derived in a different way in [24, using pointer states in string theory space.
} 
This growth in the entropy is accompanied by growth in the scale factor of the Universe, as can be seen by considering the time-dependence of the space-time volume element $V$ :

$$
\partial_{\tau} V=\partial_{\tau}\left(\int d^{D} X \sqrt{G} e^{-\Phi}\right)=\partial_{\tau}\left(e^{Q^{2} \tau} \int d^{D} X \sqrt{G}\right)=\left(Q^{2}+\partial_{\tau}\left(Q^{2}\right)\right) V
$$

The second term in (53) is $\propto O\left[\left(\beta^{i}\right)^{2}\right]$, and can be removed in an appropriate renormalization scheme. For (approximately) constant $Q$, the volume element (53) indeed grows (approximately) exponentially : $V \propto e^{Q t}$.

In this scenario, inflation occurs without an associated inflaton field, whose rôle is taken over by the Liouville time variable. Inflation is a quantum effect, as in Starobinsky's proposal [10]. However, our scenario is formulated in terms of quantum fluctuations on the world sheet.

To gain some impression how this scenario for string inflation might work in a specific model, consider the $S L(2, R) / U(1)$ model of a stringy black hole [25] with central charge $c=\frac{3 k}{k-1}-1$, for which the Friedman-Robertson-Walker line element

$$
d s^{2}=d t^{2}-R(t)^{2} d r^{2} \quad: \quad R^{2}(t)=k(t)-2
$$

where the renormalized level parameter $k(t)$ is allowed to run as in (18). It is easy to check that $R(t)$ exhibits an exponentially-growing Jeans instability [26, 27] if

$$
2 Q(k(t)-2)>\frac{k-1}{k}|\dot{k}(t)|
$$

which is the analogue of the slow rollover condition in this model.

We conclude with a discussion of density fluctuations in our scenario. Using (12) in the estimate (36), we find

$\partial_{\tau}(\delta E)^{2}=-<<\left[\left(\frac{1}{3} \delta C+\frac{1}{4} \beta^{i} G_{i j} \beta^{j}\right) \partial_{\tau} \delta C\right]>>=-<<\left[\left(\frac{1}{3} \delta C+\frac{1}{4} Q^{2}+\frac{1}{4}\left(\left(\dot{\lambda}^{\prime}\right)^{i}\right)^{2}\right) \partial_{\tau} \delta C\right]>>$

where $\delta C=C-25$, the $Q^{2}$ term on the right-hand-side is associated with graviton fluctuations in inflationary backgrounds (53), upon taking into account some properties [19] of the Quantum Zamolodchikov metric $G_{i j}$ in theory space in the class of renormalization schemes appropriate for inflation, which imply that $<<$ $\beta^{i} G_{i j} \beta^{j}>>=Q^{2}$. The $\left(\lambda^{\prime}\right)^{i}$ in (56) denote the remaining matter fields. Using the fact that $\left(\dot{\lambda}^{\prime}\right)^{i}$, being a $\beta$-function of a renormalizable coupling, does not depend explicitly on the scale $\tau$, we may cast (56) in the form

$$
\partial_{\tau}(\delta E)^{2}=<<-2 \partial_{\tau}\left[\frac{1}{3} \delta C+\frac{1}{4}\left(\left(\dot{\lambda}^{\prime}\right)^{i}\right)^{2}\right]^{2}+\frac{1}{8} \partial_{\tau}\left(\left(\dot{\lambda}^{\prime}\right)^{i}\right)^{4}>>
$$


The first term in the right-hand side of (57) is of higher order in the $\beta^{i}$ than the second term. This follows from an analysis 19 of the temporal evolution of the string density, which is constant in time during inflation [28]. Hence we estimate:

$$
(\delta E)^{2} \simeq \frac{1}{8}<<\left(\left(\dot{\lambda}^{\prime}\right)^{i}\right)^{4}>>+\ldots
$$

where the ... represent higher-order terms originating during our string inflationary epoch, as well as any other possible contributions to $(\delta E)^{2}$.

We identify $(\delta E)^{2}$ with the usual r.m.s. energy-density fluctuation $(\delta \rho)^{2}$. To estimate $\frac{\delta \rho}{\rho}$ as observed, for example, by COBE [29], we recall [1, 2] that the quantity $\delta \rho /(\rho+p)$, with $p$ the pressure, is gauge-invariant and remains constant while it is beyond the horizon. In our approach

$$
\rho+p \simeq<<\left(\dot{\lambda}^{i}\right)^{2}>>\simeq Q^{2}+<<\left(\left(\dot{\lambda}^{\prime}\right)^{i}\right)^{2}>>
$$

during inflation, and we expect $Q^{2}>><<\left(\left(\dot{\lambda}^{\prime}\right)^{i}\right)^{2}>>$ as our formulation of the conventional slow roll-over condition [1, 2], leading to the estimate :

$$
\left(\frac{\delta \rho}{\rho}\right)_{C O B E} \simeq \frac{1}{2 \sqrt{2}} \frac{<<\left(\left(\dot{\lambda}^{\prime}\right)\right)^{2}>>}{Q^{2}}=\frac{1}{2 \sqrt{2}}<<\left(\frac{d\left(\lambda^{\prime}\right)^{i}}{d t}\right)^{2}>>
$$

Formally we expect $<<\left[\frac{d\left(\lambda^{\prime}\right)^{i}}{d t}\right]^{2}>>\simeq O\left[M_{s}^{4}\right]$, where $M_{s}$ is the string unification scale [30]: $M_{s} \leq \frac{1}{10}$ Planck units. We therefore expect

$$
\left(\frac{\delta \rho}{\rho}\right)_{C O B E} \leq 10^{-4}
$$

but are not yet in a position to make more detailed estimates, which would require an exact model of the particle theory effective below the Planck scale.

\section{Acknowledgements}

The work of N.E.M. is supported by a EC Research Fellowship, Proposal Nr. ERB4001GT922259 . That of D.V.N. is partially supported by DOE grant DEFG05-91-GR-40633. 


\section{References}

[1] A.H. Guth, Phys. Rev. D23 (1981), 347; Phase Transitions in the Embryo Universe, Phil. Trans. Roy. Soc. Lond. A307 (1982), 34;

A.A. Starobinski, Phys. Lett. 117B (1982), 175;

see also D. Kazanas, Astrophysics J. 241 (1980), L59.

[2] A.D. Linde, Phys. Lett. 108B (1982), 389 ; Phys. Lett. 116B (1982), 177; ibid 335 ;

A. Albrecht and P. Steinhardt, Phys. Rev. Lett. 48 (1982), 220;

A. Vilenkin, Phys. Rev. D27 (1983), 2848.

[3] J. Ellis, K. Enqvist, D.V. Nanopoulos and M. Quiros, Nucl. Phys. B277 (1986), 231 ;

[4] A sampling of papers includes:

S. Kalara and K. Olive, Phys. Lett. B218 (1989), 148 ;

G. Veneziano, Mod. Phys. Lett. A6 (1991), 1993;

M.C. Bento, O. Bertolami and P. M. Sá, Phys. Lett. B262 (1991), 11; Mod. Phys. Lett. A7 (1992), 911.

M. Gasperini and G. Veneziano, Mod. Phys. Lett. A8 (1993), 3701;

hardly a day goes by without a new paper on this subject. Yesterday's paper was T. Damour and A. Vilenkin, IHES/Tufts preprint hep-th/9403149.

[5] I. Antoniadis, C. Bachas, J. Ellis and D.V. Nanopoulos, Phys. Lett. B211 (1988), 393; Nucl. Phys. B328 (1989), 117; Phys. Lett. B257 (1991), 278.

[6] L. Abbott, E. Fahri and M. Wise, Phys. Lett. B117 (1982), 29.

[7] A.B. Zamolodchikov, JETP Lett. 43 (1986), 730; Sov. J. Nucl. Phys. 46 (1987), 1090

A.A. Tseytlin, Phys. Lett. 178B (1986);

N.E. Mavromatos and J.L. Miramontes, Phys. Lett. B212 (1988);

N. Mavromatos, Phys. Rev. D39 (1989), 1659.

[8] N.E. Mavromatos and J.L. Miramontes, Phys. Lett. B226 (1989), 291.

[9] J. Ellis, N.E. Mavromatos and D.V. Nanopoulos, Phys. Lett. B293 (1992), 37;

Mod. Phys. Lett. A10 (1995), 425;

preprint CERN-TH.6897/93, ENS-LAPP-A427-93, CTP-TAMU-30/93; ACT10/93 (1993); hep-th/9305117; 
preprint CERN-TH.7195/94, ENS-LAPP-A-463/94, ACT-5/94, CTP-TAMU13/94, lectures presented at the Erice Summer School, 31st Course: From Supersymmetry to the Origin of Space-Time, Ettore Majorana Centre, Erice, July 4-12 1993 ; hep-th/9403133, to be published in the proceedings (World Sci. );

for a review see also D.V. Nanopoulos, Riv. Nuov. Cim. 17, No 10 (1994), 1.

[10] A. Starobinsky, Phys. Lett. B91 (1980), 99.

[11] J. Bardeen and G. Bublik, Class. Quantum Grav. 4 (1987), 573;

A.D. Linde and A. Mezhlumian, Phys. Lett. B307 (1993), 25;

A.Linde, D. Linde, and A. Mezhlumian, Phys. Rev. D49 (1994), 1783.

[12] F. David, Mod. Phys. Lett. A3 (1988), 1651;

J. Distler and H. Kawai, Nucl. Phys. B321 (1989), 509;

N.E. Mavromatos and J.L. Miramontes, Mod. Phys. Lett. A4 (1989), 1847.

[13] C. Schmidhuber, Nucl. Phys. B404 (1993), 342.

[14] N. Seiberg, Progr. Theor. Physics Suppl. 102 (1990), 319.

[15] C. Schmidhuber and A.A. Tseytlin, Nucl. Phys. B426 (1994), 187;

H. Dorn, HU-Berlin-IEP-94-21 (1994) preprint; hep-th/9410084.

[16] R. M. Santilli, Hadronic J. 1, 223 , 574 and 1279 (1978); Foundations of Theoretical Mechanics, Vol. I (1978) and II (1983) (Springer-Verlag, Heidelberg-New York);

For an application of this approach to dissipative statistical systems see: J. Fronteau, A. Tellez-Arenas and R.M. Santilli, Hadronic J. 3 (1979), 130;

J. Fronteau, Hadronic J. 4 (1981), 742.32 (1991), 142;

[17] M.B. Green, J.H. Schwarz and E. Witten, String Theory, Vol. I and II (Cambridge Univ. Press 1986).

[18] S. Hojman and C. Shepley, J. Math. Phys. 32 (1991), 142;

F. Pardo, J. Math. Phys. 30 (1989), 2054.

[19] J. Ellis, N.E. Mavromatos and D.V. Nanopoulos, to appear.

[20] J. Ellis, N.E. Mavromatos and D.V. Nanopoulos, CERN and Texas A \& M Univ. preprint CERN-TH. 6755/92; ACT-24/92;CTP-TAMU-83/92; hep-th/9212057.

[21] S. Das, G. Mandal and S. Wadia, Mod. Phys. Lett. A4 (1989), 745 ;

D. Friedan, unpublished, as quoted in T. Banks and E. Martinec, Nucl. Phys. B294 (1987), 733. 
[22] Y.R. Shen, Phys. Rev. 155 (1967), 921;

A.S. Davydov and A. A. Serikov, Phys. Stat. Sol. B51 (1972), 57;

B.Ya. Zel'dovich, A.M. Perelomov, and V.S. Popov, Sov. Phys. JETP 28 (1969), 308 ;

For a review see : V. Gorini et al., Rep. Math. Phys. Vol. 13 (1978), 149.

[23] J. Binney, N. Dowrick, A. Fisher and M. Newman, The Theory of Critical Phenomena (Clarendon Press Oxford 1992).

[24] J. Ellis, N.E. Mavromatos and D.V. Nanopoulos, Proc. First International Conference on 'Phenomenology of Unification from Present to Future', Rome, 'La Sapienza' University, March 22-26 1994 ; preprint CERN-TH.7269/94, CTPTAMU 26/94, ENSLAPP-A-474/94, hep-th/9405196.

[25] E. Witten, Phys. Rev. D44 (1991), 344.

[26] J. Ellis, N.E. Mavromatos and D.V. Nanopoulos, Proc. HARC workshop on "Recent Advances in the Superworld", The Woodlands, Texas (USA), April 1416 1993, eds. J. Lopez and D.V. Nanopoulos, (World Sci., Singapore, 1994),p. 3; preprint CERN-TH.7000/93 , CTP-TAMU 66/93 , ENSLAPP-A-445/93 , OUTP-93-26P ; hep-th/9311148.

[27] N. Sanchez and G. Veneziano, Nucl. Phys. B333 (1990), 253.

[28] N. Turok, Phys. Rev. Lett. 60 (1988), 549.

[29] G.F. Smoot et al., Ap. J. Lett. 396, L1 (1992);

E.L. Wright et al., Ap. J 420 (1994), 1;

K. Gorski et al., Ap. J. 430, L89 (1994).

[30] J. Minahan, Nucl. Phys. B298 (1988), 36;

V. Kaplunovsky, Nucl. Phys. B307 (1988), 145; erratum B382 (1992), 436;

I. Antoniadis, J. Ellis, R. Lacaze and D.V. Nanopoulos, Phys. Lett. B268 (1991), 188 ;

L. Dixon, V. Kaplunovsky and J. Louis, Nucl. Phys. B355 (1991), 649 ;

J-P. Derendinger, S. Ferrara, C. Kounnas, and F. Zwirner, Nucl. Phys. B372 (1992), 145. 